\title{
Evaluación de mapas de competencias educativas: una propuesta difusa basada en 2-tuplas
}

\author{
Diego F. Duran ${ }^{1}$, Gabriel E. Chanchí ${ }^{2}$, Jose L. Arciniegas ${ }^{1}$ \\ dduran@unicauca.edu.co,gchanchi@unimayor.edu.co,jlarci@unicauca.edu.co \\ ${ }^{1}$ Universidad del Cauca, Cll. 5 \#4-70, 190003, Popayán, Colombia. \\ ${ }^{2}$ Institución universitaria Colegio Mayor del Cauca, Cra. 7 \#2-34, 190003, Popayán, Colombia.
}

DOI: 10.17013/risti.24.22-38

\begin{abstract}
Resumen: Aunque el uso de términos lingüísticos permite a los docentes realizar una evaluación de competencias educativas de una forma natural y significativa, la evaluación de mapas de competencias puede ser una tarea imprecisa, ya que debe ser considerada una gran cantidad de información sobre las relaciones existentes entre elementos (competencias y actividades de evaluación), pesos de tales relaciones y niveles de competencia. Con el propósito de abordar este problema, este trabajo propone un modelo difuso basado en 2-tuplas lingüísticas para evaluar competencias educativas teniendo en cuenta las características de los mapas de competencias. Adicionalmente, se presenta una aplicación Java, la cual fue usada para evaluar la propuesta mediante datos reales. Los resultados obtenidos permiten concluir que la propuesta es efectiva y eficiente.
\end{abstract}

Palabras-clave: Educación, Evaluación, Lógica difusa, Mapas de competencias.

\section{Competency maps assessment: a fuzzy proposal based on 2-tuples}

\begin{abstract}
Although the use of linguistic terms enables teachers carry out educational competencies assessment in a natural and significant way, the competency maps assessment may be an imprecise task because teachers have to consider a large quantity of information related to relations among elements (i.e., competencies and assessment activities), weights of such relations and proficiency levels. In order to address this issue, this work proposes a fuzzy model for assessing competencies based on the features of competency maps by using the 2-tuples approach. Additionally, a Java application is presented, which was used to assess the proposal by means of real data. The results indicate that the proposal is quite effective and efficient.
\end{abstract}

Keywords: Assessment, Competency maps, Education, Fuzzy Logic.

\section{Introducción}

En el contexto educativo, las competencias son las diferentes habilidades que los estudiantes pueden aprender y los docentes enseñar. Éstas son de gran importancia 
porque definen criterios públicos de la calidad educativa de una institución (OCDE, 2006). Ejemplos de tal importancia son la educación basada en competencias, la cual es desarrollada en algunos países de América Latina (por ejemplo, Colombia y Perú) y la Declaración de Bolonia, en la cual el concepto de evaluación de competencias fue seleccionado como el parámetro básico para comparar la educación superior entre diferentes universidades europeas (en 29 países como España, Alemania y Francia) (Serrano, Romero \& Olivas, 2013). En esta dinámica, la evaluación de competencias es una tarea clave. Los docentes califican Actividades de Evaluación (AE) asignando Niveles de Competencia (NC) de acuerdo al desempeño del estudiante. Esta tarea puede ser realizada usando notas numéricas en el rango $[0,10]$; sin embargo, el uso de términos lingüísticos (por ejemplo, Bajo, Medio, Alto) puede ser más natural y significativo en la evaluación de algunos tipos de AE y competencias (Fung, 2013), por ejemplo, si estas involucran habilidades y capacidades observables de los estudiantes en actividades de solución de problemas.

Aunque el uso de términos lingüísticos es útil, la evaluación de competencias puede ser un proceso difícil e impreciso cuando el docente evalúa estructuras en las que se establecen relaciones entre ellas, ya que debe ser considerada una gran cantidad de información. Éste es el caso de los mapas de competencias, los cuales sirven como herramientas para observar los NC alcanzados en el currículo de un curso o programa (García \& Gairín, 2011). De acuerdo con el borrador de estándar Simple Reusable Competency Map (SRCM), los mapas de competencias están compuestos por competencias, relaciones de tipo general-específica entre éstas con etiquetas de pesos (estos ponderan la importancia de una competencia específica en el desarrollo de una general), y los NC asignados en los procesos de calificación. Con el propósito de abordar el problema mencionado, el presente artículo propone un enfoque difuso para evaluar competencias, basado en la estructura y características de los mapas de competencias usando el enfoque de 2-tuplas lingüísticas. El enfoque difuso es usado porque permite una mejor representación del lenguaje natural (Herrera, López, Alonso, Moreno, Cabrerizo \& Porcel, 2009). Adicionalmente, en este trabajo se desarrolló una herramienta Java basada en la propuesta, la cual se encarga de computar los $\mathrm{NC}$ de todas las competencias de los mapas mientras mide y presenta los tiempos de ejecución utilizados.

El resto del artículo está organizado como sigue. En la Sección 2 se presentan los conceptos introductorios y los trabajos previos relacionados con esta investigación. La Sección 3 describe el enfoque propuesto, incluyendo la construcción de mapas de competencias y del perfil del estudiante (desde el punto de vista computacional) y el proceso de cómputo de los NC. La Sección 4 presenta una evaluación de la propuesta en términos de efectividad y tiempos de ejecución, utilizando la herramienta Java desarrollada y datos reales provistos por los docentes. Finalmente, en la Sección 5 se presentan las conclusiones y trabajos futuros derivados de la presente investigación.

\section{Aspectos preliminares}

Esta sección presenta un grupo de conceptos, aspectos y trabajos relacionados con la presente investigación. Dentro de estos se encuentran: los mapas de competencias y los problemas existentes en su evaluación, los enfoques difusos, incluyendo los basados en 2-tuplas y en el principio de extensión. 


\subsection{Mapas de competencias}

Los mapas de competencias son herramientas usadas por los docentes para observar los NC alcanzados por los estudiantes en el currículo de una materia, área, programa o curso (García \& Gairín, 2011). Una buena aproximación a la estructura de un mapa de competencias es dada por el borrador de estándar SRCM (Ostyn, 2006). Éste define un modelo de información simple y reusable para mapas de competencias, para ser usado en la descripción, referencia e intercambio de datos que definen relaciones entre competencias en el contexto educativo. En el modelo de SRCM, un Grafo Acíclico Dirigido (GAD) es usado para describir los mapas, en el cual cada competencia es representada por un nodo. Dado que un GAD es una colección jerárquica de nodos, una competencia puede ser descompuesta en sub-competencias, o que tales sub-competencias contribuyen a ella. La Figura 1 muestra un fragmento de un mapa de competencias basado en el modelo de SRCM, el cual fue provisto por un docente de matemáticas que usa los estándares Básicos de Competencias de Colombia para establecer las metas de enseñanza y aprendizaje (Ministerio de Educación de Colombia, 2006). Como puede observarse, los mapas tienen las siguientes características:

- Las competencias tienen sub-elementos que pueden ser AE, e incluso, otras competencias (notar que una competencia puede ser un sub-elemento o subcompetencia de otra). Entonces, los mapas pueden estar compuestos por uno o más niveles.

- Las AE diseñadas por los docentes (exámenes orales o escritos, solución de problemas, etc.) han sido incluidas como sub-elementos de algunas competencias. En la práctica educativa, éstas son usadas por los docentes para realizar un proceso de calificación de los estudiantes, en el cual unos NC son asignados.

- La estructura establece relaciones entre sus elementos, las cuales son etiquetadas con un peso que representa la importancia de una sub-competencia en el desarrollo de una competencia de un nivel superior.

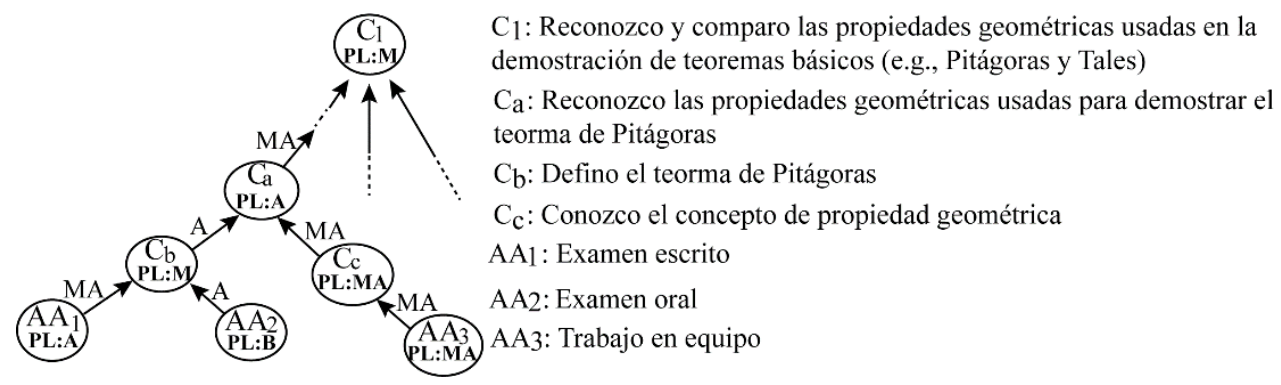

Figura 1 - Fragmento de un mapa de competencias basado en el modelo de SRCM

- Los NC han sido asignados por el docente a todos los elementos del mapa. Los docentes usualmente usan los NC asignados a los AE para computar mentalmente los de todas las competencias.

En el ejemplo presentado, pesos y NC fueron asignados de acuerdo a un grupo de siete términos lingüísticos como el siguiente: 
Como comentario final, aunque la misma estructura puede ser usada para varios estudiantes, cada mapa de competencia corresponde a un solo estudiante porque registra sus propios NC.

\subsection{Problemas en la evaluación de competencias}

De acuerdo con (Fung, 2013), el criterio del docente en un proceso de evaluación en un AE está usualmente determinado por grados numéricos de 1 a 5, o términos lingüísticos asociados con números, como en el caso de las escalas Likert (Likert, 1932), por ejemplo, "Muy Bajo" está asociado a 1, "Bajo" a 2, "Medio" a 3, "Alto" a 4, "Muy Alto" a 5. Cuando el docente observa estas posibles opciones, su cerebro procesa información del proceso de evaluación y elige una para asignar un NC que describa su valor de percepción. Sin embargo, existen algunos aspectos que pueden entorpecer este proceso, tales como la incertidumbre, restricciones, y el vago conocimiento que pueda tener el docente. Incluso, es muy difícil para él asegurar que los criterios numéricos sean igualmente aplicados al evaluar a todos los estudiantes. De acuerdo con (Montero, Alias, Badía, Fonseca \& Vicent, 2014), estos problemas se dan especialmente si la AE involucra habilidades y capacidades observables en actividades de solución de problemas (por ejemplo, una mesa redonda, experimentos químicos, etc.) o cuando las competencias evaluadas son de una naturaleza subjetiva, tales como la competencia "trabajo en equipo". Con el propósito de resolver estos problemas, en trabajos como (Fung, 2013), (Zhaoa \& Yueb, 2012) y (Massanet, Riera, Torrens \& Herrera, 2014), la evaluación es realizada usando términos lingüísticos como los mostrados en la expresión (1), los cuales hacen el juicio del docente (y de cualquier experto en determinado contexto) más confiable y consistente.

Asumiendo que los docentes usan términos lingüísticos para llevar a cabo procesos de evaluación de AE, la evaluación de mapas puede ser una tarea imprecisa debido a que éstos deben considerar una gran cantidad de información lingüística. En consecuencia, los NC pueden ser determinados incorrectamente, especialmente, en los niveles superiores de los mapas.

\subsection{Enfoque lingüístico difuso}

Hay situaciones en las cuales no es apropiado el uso de una evaluación cuantitativa. En estos casos, la utilización de un enfoque lingüístico puede ser más conveniente, en el cual, son usados un conjunto de términos como los mostrados en la expresión (1). De acuerdo con (Herrera \& Martínez, 2000), una forma de generar un conjunto lingüísticos consiste en distribuir los términos en una escala con un orden bien establecido. Un ejemplo es $S=\left\{s_{\mathrm{o}}: N, s_{1}: M B, s_{2}: \mathrm{B}, s_{3}: M, s_{4}: A, s_{5}: M A, s_{6}: T\right\}$. Como puede observarse, un grupo de índices han sido incluidos en la expresión (1). De acuerdo con el criterio de expertos que participaron en esta investigación, éste es el conjunto más apropiado en la práctica educativa en cuanto a términos y granularidad (esto es, la cardinalidad del conjunto de términos).

Por otro lado, en este trabajo son usadas las funciones de pertenencia triangulares (ver la Figura 2). La semántica de los siete términos se define de la siguiente forma: 


$$
\begin{aligned}
& N=(0,0,0.17), M B=(0,0.17,0.33), B=(0.17,0.33,0.5), M=(0.33,0.5,0.67) \\
& A=(0.5,0.67,0.83), M A=(0.67,0.83,1), T=(0.83,1,1)
\end{aligned}
$$

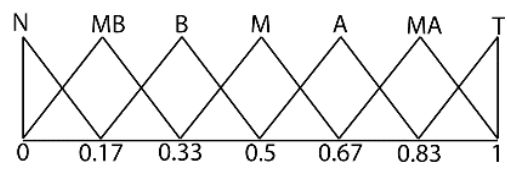

Figura 2 - Un grupo de siete términos lingüísticos y su semántica

Dentro del enfoque lingüístico difuso, se encuentran enfoques computacionales como el basado en 2-tuplas y el basado en el principio de extensión, los cuales son importantes para la presente propuesta.

\subsection{Enfoque computacional basado en 2-tuplas}

Este enfoque está basado en el concepto de translación simbólica, la cual se define como sigue: sea un conjunto de términos lingüísticos, si un método simbólico agregando información lingüística obtiene un valor $\beta \in[0, g], \mathrm{y} \beta \notin\{0, \ldots, g\}$ entonces una función de agregación $\left(\operatorname{app}_{2}().\right)$ es usada para expresar el índice del resultado en $S$. La translación simbólica de un término lingüístico $s_{i}$ es un valor numérico evaluado en [-0.5,0.5) que apoya la "diferencia de información" entre una cantidad de información $\beta \in[0, g]$ obtenida después de una operación de agregación simbólica y el valor más cercano en $\{0, \ldots, g\}$ que indica el índice del término lingüístico más cercano en $S(i=\operatorname{round}(\beta))$. Este enfoque representa la información lingüística por medio de 2-tuplas $\left(s_{i}, \alpha_{i}\right), s_{i} \in S$ y $\alpha_{i} \in$ $[-0.5,0.5)$, siendo $s_{i}$ una representación de la etiqueta lingüística centro de la información y $\alpha_{i}$ un valor numérico que expresa el valor de la translación desde el resultado original $\beta$ a la etiqueta del índice más cercana $i$ en el conjunto de términos lingüísticos $s_{i}$, esto es, la translación simbólica. Además, se define un conjunto de funciones de transformación entre términos lingüísticos y 2-tuplas, y entre valores numéricos y 2-tuplas. Las 2-tuplas que expresan la información equivalente a $\beta$ es obtenida con las siguientes funciones:

$$
\begin{gathered}
\Delta:[0, g] \rightarrow S x[-0.5 \cdot 0.5] \\
\Delta(\beta)=\left\{\begin{array}{c}
s_{i}, i=\operatorname{round}(\beta) \\
\alpha=\beta-i, \alpha \in[-0.5,0.5)
\end{array}\right.
\end{gathered}
$$

Donde round(.) es la operación de redondeo común, $s_{i}$ tiene la etiqueta más cercana a $\beta$ y $\alpha$ es el valor de la translación simbólica. Como un comentario importante, la conversión de un término lingüístico a una 2-tupla consiste en añadir un valor cero de la siguiente forma:

$$
s_{i} \in \mathrm{S} \Rightarrow\left(s_{i}, \mathrm{O}\right)
$$




\subsection{Enfoque computacional basado en el principio de extensión}

El principio de extensión ha sido introducido para generalizar operaciones matemáticas numéricas a los conjuntos difusos. Para esto, se usan operadores de agregación lingüísticos, los cuales actúan de acuerdo con:

$$
S^{n} \stackrel{\tilde{F}}{\rightarrow} F(\mathcal{R}) \stackrel{a p p_{1}(\cdot)}{\rightarrow} S
$$

Donde $S^{n}$ simboliza el producto cartesiano $n$ de $S, \widetilde{F}$ es un operador de agregación basado en el principio de extensión, $F(R)$ es el conjunto de conjuntos difusos sobre el conjunto de números reales $R, \operatorname{app}_{1}: F(R) \rightarrow S$ es una función de aproximación lingüística que retorna una etiqueta del conjunto de términos lingüísticos Scuyo significado es el más cercano al número difuso no etiquetado obtenido y $S$ es el conjunto de términos inicial.

\subsection{Trabajos relacionados}

Existen trabajos en la literatura centrados en evaluar competencias usando enfoques lingüísticos difusos. En (Pengshun \& Jianguo, 2012) se presenta una metodología para evaluar competencias laborales de los estudiantes. Tal propuesta aplica un método de evaluación difuso basado en una estructura de competencias profesionales, la cual es una estructura en árbol de tres niveles con competencias pre-definidas. En (Serrano, Romero \& Olivas, 2013) se propone un enfoque difuso para recomendar actividades educativas. Inicialmente, se define un modelo basado en 2-tuplas que posibilita la evaluación lingüística de competencias sobre una estructura compuesta de un grupo de competencias y actividades. La estructura define que cada competencia está relacionada con un grupo de actividades. Después, se define un modelo de recomendación que usa un filtro basado en los resultados de la evaluación. En (Zhaojun, Xiao \& Xiaoqing, 2010) se presenta un modelo para evaluar competencias educativas basado en un enfoque difuso. En un caso de estudio, el modelo es aplicado a un sistema de índices de competencias para vendedores, el cual es una estructura en árbol compuesta por 3 niveles. En (Jia \& Zhao, 2010) se propone un método de evaluación de competencias básicas para empresas. Éste se basa en la entropía y los números difusos triangulares, posibilitando una evaluación objetiva de empresas. Éste puede ser útil en un proceso de decisión, en el cual un grupo de empresas son comparadas entre sí de acuerdo al criterio de expertos. En (Zhang, 2012) se propone un método de decisión basado en operadores de agregación. Éste usa el modelo de 2-tuplas para agregar los desempeños de las alternativas medidas por un grupo de evaluadores. El resultado de la agregación es usado para tomar una decisión del grupo de alternativas.

Todos los trabajos relacionados revelan la importancia de la evaluación de competencias y desempeños por medio de términos lingüísticos. Para este propósito, varios modelos y enfoques han sido propuestos para facilitar el proceso de evaluación con un alto nivel de efectividad. Sin embargo, no hay evidencia de investigaciones basadas en la estructura y características de los mapas de competencias (ver la Sección 2.2). Adicionalmente, aunque todas las propuestas son efectivas, no ha sido realizada una evaluación de su viabilidad en términos de eficiencia. 


\section{Propuesta de evaluación de competencias}

La presente propuesta computa los $\mathrm{NC}$ de todos los elementos de los mapas de competencias de forma cualitativa, a partir de los alcanzados por los estudiantes en las AE. Para este propósito, el modelo considera las características de los mapas, con respecto a su estructura, tipos de elementos y pesos (ver la Sección 2.1). Las próximas subsecciones inicialmente describen metodológicamente cómo son construidos los mapas de competencias y los perfiles de los usuarios desde el punto de vista computacional. Posteriormente, se describe el proceso basado en 2-tuplas para el cómputo de los NC de todo el mapa.

\subsection{Fase A: Representación de la estructura del mapa de competencias}

Los currículos educativos están basados en un grupo de competencias generales establecidas por un ente gubernamental (por ejemplo, el Ministerio de Educación Nacional a través de los Estándares básicos de competencias) o por la dirección general de una institución (por ejemplo, una escuela o universidad). Tales competencias deben ser alcanzadas por los estudiantes durante el ciclo escolar. Debido a la universalidad inherente a las competencias generales, a partir de éstas pueden definirse un grupo de sub-competencias, relacionadas unas con otras con diferentes pesos, de tal forma que al final se consigue establecer una guía para los docentes de cómo alcanzar las metas educativas. A partir de esta dinámica se definen unos vectores que en conjunto constituyen la estructura de los mapas:

1. Dado que una competencia está relacionada con sus sub-elementos, los cuales pueden ser otras competencias o $\mathrm{AE}$ según sea el caso, entonces una competencia se define por medio del vector $\left\{C_{1}, C_{2}, \ldots, C_{j} \ldots, C_{k}\right\}$.

2. Dado que los pesos están asociados a la relación entre $C_{i}$ relacionados y su subelemento $C_{j}$, éstos son representados por el vector $\left\{P_{i 1}, P_{i 2}, \ldots, P_{i j} \ldots ., P_{i k}\right\}$.

En este punto, la estructura del mapa puede ser representada por medio de los vectores descritos. Sin embargo, aún falta incorporar los NC del estudiante antes de iniciar el proceso de cómputo. Esta situación es tratada en las siguientes sub-secciones.

\subsection{Fase B: Construcción del perfil del estudiante}

Cuando un estudiante realiza una $\mathrm{AE}$, el docente califica su desempeño asignando un NC lingüístico. Por consiguiente, el perfil del estudiante está compuesto por un grupo de $\mathrm{NC}$ alcanzados en cada $\mathrm{AE} A E_{j}$ o competencia $C_{j}$. Entonces, el grupo de NC correspondientes a los sub-elementos de $C_{i}$, es representado por el vector $\left\{N C_{1}, N C_{2}, \ldots, N C_{j}, \ldots, N C_{k}\right\}$. En ciertos momentos del ciclo escolar es necesario conocer las competencias alcanzadas o adquiridas por el estudiante. Para este propósito, en este trabajo se propone un proceso de cómputo basado en 2-tuplas, el cual es expuesto en las próximas sub-secciones.

Ejemplo 1: construcción de vectores. La Figura 3 presenta un ejemplo de un mapa de competencias de tres niveles. De acuerdo al modelo de vectores descrito anteriormente, 
la estructura del mapa se representa por medio de los vectores $C_{1}=\left\{C_{2}, C_{3}\right\}$, $C_{2}=\left\{A E_{1}, A E_{2}\right\}, C_{3}=\left\{A E_{2}, A E_{3}\right\}, P_{1}=\{A, M A\}, P_{2}=\{M, M A\}$ y $P_{3}=\{A, B\}$. Adicionalmente, el perfil del estudiante es representado por los vectores $N C_{2}=\{B, A\}$ y $N C_{3}=\{A, M A\}$.

\subsection{Fase C: Cómputo de los Niveles de Competencia}

Con el propósito de computar los NC de todos los elementos del mapa, el modelo propuesto agrega los NC del perfil del estudiante considerando los vectores de la estructura del mapa. Todas las sub-fases del proceso, las cuales son una adaptación del modelo basado en 2-tuplas, son explicadas a continuación.

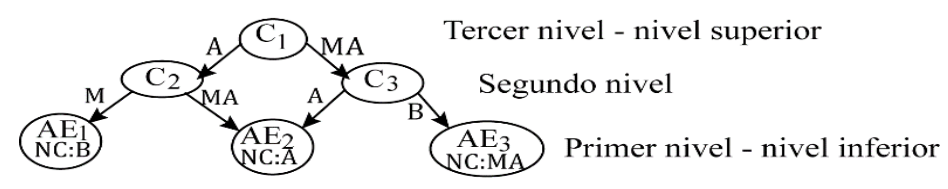

Figura 3 - Ejemplo de un mapa de competencias

\section{Sub-fase C-1: Construcción de matrices}

En esta sub-fase los vectores de las fases A y B son utilizados para construir el modelo matricial mostrado en la Figura 4, el cual posee las características pertinentes para la aplicación de operadores difusos de agregación. Este par de matrices son construidas por cada nivel del mapa. La primera, llamada Matriz de Niveles de Competencia (MNC), organiza las competencias en las columnas y sus sub-elementos en las filas. Los elementos de MNC son los NC $N C_{i j}$ alcanzados por el estudiante en la $A E_{j}$ o $C_{j}$, siendo estos subelementos de $C_{i}$. La segunda matriz, llamada Matriz de Pesos (MP), también organiza las competencias en las columnas y las AE o competencias en las filas. Sin embargo, en este caso cada elemento de MP corresponde al peso $P_{i j}$ de la relación entre $A E_{j}$ o $C_{j}$ y $C_{i}$. En ambos casos, si no existe una relación entre una columna y una fila, el valor del elemento correspondiente es cero. En la Figura 4 se puede apreciar que las matrices organizan las $\mathrm{AE}$ o las competencias según sea necesario.

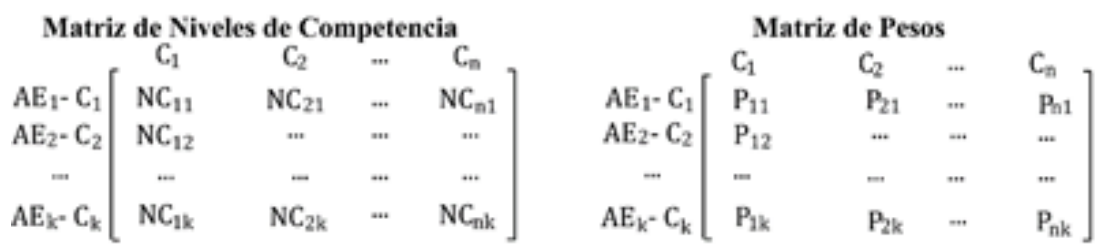

Figura 4 - Matrices de Niveles de Competencia y Pesos

Por otra parte, de acuerdo con el procesamiento de palabras llevado a cabo en la lógica difusa (ver Secciones 2.4 y 2.5), se da que en la agregación se obtiene inicialmente un valor numérico, el cual es posteriormente analizado para asignarle un valor lingüístico 
(el cual es considerado como resultado final de la agregación). Para diferenciarlos, en lo que sigue de este trabajo son nombrados como Valor de Agregación Numérico (VAN) y valor de agregación lingüístico (VAL). Considerando que los NC computados para cierto nivel influyen en el cómputo de los NC de niveles superiores, en el presente enfoque se proponen dos posibilidades para realizar la agregación:

- Posibilidad P1. La agregación en todos los niveles es realizada usando los VAL del nivel inmediatamente inferior. En este caso, MNC y MP se compone de términos lingüísticos.

- Posibilidad P2. La agregación a partir del tercer nivel es realizada usando los VAN del nivel inmediatamente inferior (notar que en la agregación del segundo nivel son usados los NC del primer nivel, los cuales son términos lingüísticos). En este caso, MNC se compone de valores numéricos mientras que PM lo hace de términos lingüísticos.

El uso de una u otra posibilidad depende del criterio del usuario. De cualquier forma, ambas tienen implicaciones en el modelo, las cuales son tratadas en las siguientes sub-fases.

\section{Sub-fase C-2: Conversión de términos lingüísticos a 2-tuplas}

En esta sub-fase, cada término lingüístico que hace parte de las matrices es convertido a 2-tuplas. De acuerdo con la expresión (4), un valor de cero debe ser añadido a cada término con el propósito de hacer translaciones simbólicas. En caso de hacer uso de la segunda de las posibilidades presentadas en la sub-fase C-1, la conversión es aplicada únicamente sobre la MP.

Ejemplo 2: construcción de matrices. Considerando el Ejemplo 1, la Figura 5 presenta las matrices MNC y MP para el segundo nivel del mapa. Notar que todos los términos lingüísticos han sido convertidos a 2-tuplas.

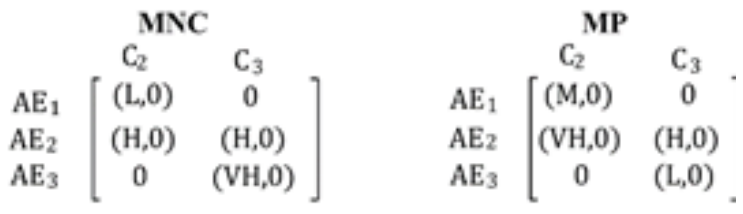

Figura 5 - MNC y MP para el Ejemplo 2.

\section{Sub-fase C-3: Agregación}

Una vez organizados los $\mathrm{NC}$, ya sea de las AE o de las competencias de cierto nivel del mapa, se computan los NC de todos los elementos del nivel inmediatamente superior. Para esto, se proponen dos diferentes procesos de agregación para cada una de las posibilidades establecidas en la sub-fase C-1.

Agregación para la posibilidad P1. Se establece el uso del Operador de Media Ponderada (WAO por su significado en inglés) para 2-tuplas descrito en (Herrera \& Martínez, 2000): 


$$
\bar{x}^{e}=\Delta\left(\frac{\sum_{i=1}^{n} \beta_{i}^{*} w_{i}}{\sum_{i=1}^{n} w_{i}}\right)
$$

Este operador permite a diferentes valores de tener diferentes importancias en la naturaleza de la variable $x$. Por lo tanto, cada valor de $x_{i}$ tiene un peso asociado $w_{i}$ que indica su importancia en la naturaleza de la variable. En términos de competencia, este operador permite agregar diferentes $\mathrm{NC}$ alcanzados en $\mathrm{AE}$ o competencias considerando su importancia para la competencia de la cual son sub-elementos. La función $\Delta$ es tratada en la Sección 2.4. Los valores de $\beta$ para todos los índices de los términos lingüísticos son definidos en la Figura 6. Por ejemplo, los valores de $\beta$ para B, A y MA son 2, 4 y 5 respectivamente.

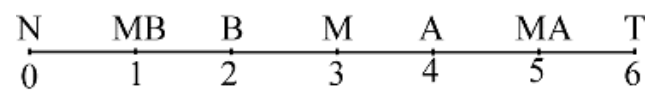

Figura 6 - Distribución de índices para los términos lingüísticos

Agregación para la posibilidad P2. Se establece el uso del operador WAO definido en la expresión (5) con una variación de la naturaleza de $\beta$. En este caso, al aplicar el WAO para cierta competencia, los valores de $\beta$ usados corresponden a los VAN computados para sus sub-elementos.

Ejemplo 3: agregación de 2-tuplas. Considerando el ejemplo 2, en este ejemplo son calculados los NC para las competencias $C_{2}$ y $C_{3}$ usando la expresión (5):

$$
N C_{C 2}=\Delta\left(\frac{2^{*} 3+4^{*} 5}{3+5}\right)=\Delta(3.25)
$$

Siguiendo un proceso similar, se obtiene $N C_{C_{3}}=\Delta$ (4.33). La función es utilizada para asignar términos lingüísticos en la siguiente sub-fase.

\section{Sub-fase C-4: Asignación del término lingüístico}

En esta sub-fase, la función interpreta los VAN para asignar los correspondientes términos lingüísticos. De acuerdo con el modelo basado en 2-tuplas, la función genera un par de 2-tuplas $\left(S_{a}, \alpha_{a}\right)$ y $\left(S_{b}, \alpha_{b}\right)$ que corresponden al término más cercano inferior y superior del VAN. $S_{a}$ es asignado si $\left|\alpha_{a}\right|<\left|\alpha_{b}\right|$, mientras que $S_{b}$ es asignado si $\left|\alpha_{b}\right|<\left|\alpha_{b}\right|$. Ejemplo 4: agregación y asignación de términos lingüísticos. Teniendo en cuenta que la competencia $C_{1}$ está en el tercer nivel del mapa, el cómputo de su NC puede ser realizado de acuerdo a las dos posibilidades establecidas anteriormente: 
Considerando con la posibilidad P1. La Figura 7 presenta las matrices para el nivel superior del mapa. NCM está compuesta por los VAL computados para $C_{2}$ y $C_{3}$.

Entonces el NC para $C_{1}$ es calculado como sigue:

$$
N C_{C_{1}}=\Delta\left(\frac{3^{*} 4+4^{*} 5}{4+5}\right)=\Delta(3.55)
$$

Considerando la posibilidad P2. La Figura 8 muestra las matrices para el nivel superior del mapa. NCM está compuesta por los VAN computados para $C_{2}$ y $C_{3}$. Entonces el NC para $C_{1}$ es calculado como sigue:

$$
\begin{aligned}
& N C_{C 1}=\Delta\left(\frac{3.25 * 4+4.33^{*} 5}{4+5}\right)=\Delta(3.85) \\
& \begin{array}{cc}
\mathbf{M N C} & \mathbf{M P} \\
\mathrm{C}_{1} & \mathrm{C}_{1} \\
\mathrm{C}_{2}\left[\begin{array}{c}
(\mathrm{M}, \mathrm{O}) \\
\mathrm{C}_{3}[(\mathrm{H}, 0)
\end{array}\right. & \mathrm{C}_{2}[(\mathrm{H}, 0)] \\
\mathrm{C}_{3}[(\mathrm{VH}, 0)]
\end{array}
\end{aligned}
$$

Figura 7 - MNC y MP para el nivel superior usando la posibilidad P1

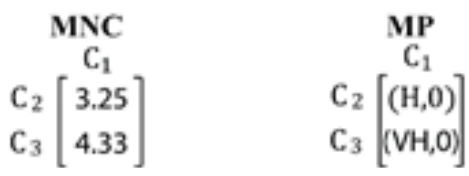

Figura 8 - MNC y MP para el nivel superior usando la posibilidad P2

Para la asignación de los términos lingüísticos, se tiene que $N C_{C 2}=\Delta(3.25)$. Dado que 3.25 está ubicado entre los términos $\mathrm{M}$ y A, la función da como resultado las 2-tuplas $(M, 0.25)$ y $(M,-0.75)$. Como $|0.25|<|-075|$, entonces el término asignado a $C_{2}$ es M. En un proceso similar se da que el término asignado para $C_{3}$ y $C_{1}$ es A en ambos casos. Sin embargo, notar que los VAN para $C_{1}$ fueron 3.55 y 3.85 para cada una de las posibilidades. Aunque en ambos casos el término asignado es A, el primer valor se ubica en un punto medio entre $\mathrm{M}$ y A, mientras que el segundo es claramente cercano a A. Esta diferencia de resultados se da porque usando la primera posibilidad hay una pérdida de información nivel por nivel, resultado de la función de redondeo inherente a la función $\Delta$. En cambio, con el uso de la segunda posibilidad, el cómputo de los NC de un nivel considera todos los NC de los niveles inferiores, por lo cual no hay pérdida de información. Así, es probable que en mapas complejos (con más elementos y niveles), el término lingüístico asignado difiera entre una y otra posibilidad. Aunque son claras las consecuencias dadas en el uso de cada una, la elección de una de las posibilidades dependerá del criterio del docente. 


\section{Evaluación}

Esta sección presenta inicialmente un grupo de experimentos realizados para evaluar la propuesta usando datos reales. Posteriormente se presentan los resultados obtenidos.

\subsection{Visión general}

Los experimentos diseñados para dar respuesta a las siguientes preguntas de investigación: (1) ¿Cuál es la efectividad de la propuesta?; (2) ¿Qué ventajas provee la propuesta en términos de tiempo de ejecución en comparación con el enfoque basado en el principio de extensión?

Con el propósito de responderlas, se desarrolló una herramienta software soportada en el lenguaje Java, la cual fue útil para realizar los experimentos. Ésta ofrece dos modos de operación para computar los NC de las competencias (ver Figura 9): el Modo 1 está basado en la presente propuesta y el Modo 2 está basado en el principio de extensión. Los datos que describen la estructura de los mapas y los NC de los estudiantes han sido adaptados al formato JSON y almacenados en una base de datos NoSQL MongoDB. La aplicación computa los NC de todos los elementos de los mapas, presentando tanto los VAL como los VAN. Adicionalmente, presenta los tiempos de ejecución medidos para cada mapa. Como puede observarse, la aplicación provee los datos necesarios para evaluar la propuesta.

\subsection{Métricas}

La métrica definida en la expresión (6) fue usada para dar respuesta a la primera pregunta de investigación, la cual se define como el porcentaje de NC dados por la aplicación que son iguales a los proveídos por los docentes. También, puede ser considerada como el porcentaje de aciertos de la propuesta.

$$
A=\frac{N_{\text {hits }}}{T} * 100 \%
$$

Donde $N_{\text {hits }}$ es el total de aciertos y $T$ es el total de NC evaluados. Con respecto a la segunda pregunta de investigación, el tiempo de ejecución de la aplicación es una variable que depende de varios factores, tales como los recursos propios del computador o las tareas paralelas realizadas por él al realizar los experimentos. Con el propósito de reducir la variabilidad de las medidas del tiempo de ejecución, se ha calculado un valor promedio $t_{a}$ de cien medidas para cada experimento $a$, de acuerdo con:

$$
t_{a}=\frac{\sum_{i=1}^{100} t_{i a}}{100}
$$

Donde $t_{i a}$ es la $i$-ésima medida de tiempo tomada para el experimento $a$.

\subsection{Experimentos y resultados}

Con el propósito de evaluar la presente propuesta, se hizo uso de los datos de 75 mapas, los cuales fueron construidos y provistos por un grupo de docentes basándose en los Estándares Básicos de Competencias en Matemáticas para Colombia. A fin de explicar 
de forma clara los experimentos y resultados alcanzados, se ha decidido en adelante diferenciar lo que se constituye como la estructura del mapa de lo que representa el mapa de competencias en sí. Con estructura del mapa se refiere al grupo de competencias, sus relaciones y pesos, mientras que el mapa de competencias adiciona a la estructura del mapa los NC alcanzados por cada estudiante en las AE y competencias. Así, un grupo de mapas de competencias (uno por estudiante) puede ser el resultado de evaluar una misma estructura. Los 75 mapas usados en la evaluación obedecen a 15 diferentes estructuras, esto es, 5 mapas por estructura. Estos incluyen los $\mathrm{NC}$ alcanzados por un grupo de estudiantes en todas las AE y competencias del mapa, razón por la cual son propicios para dar cuenta de la efectividad de la propuesta.
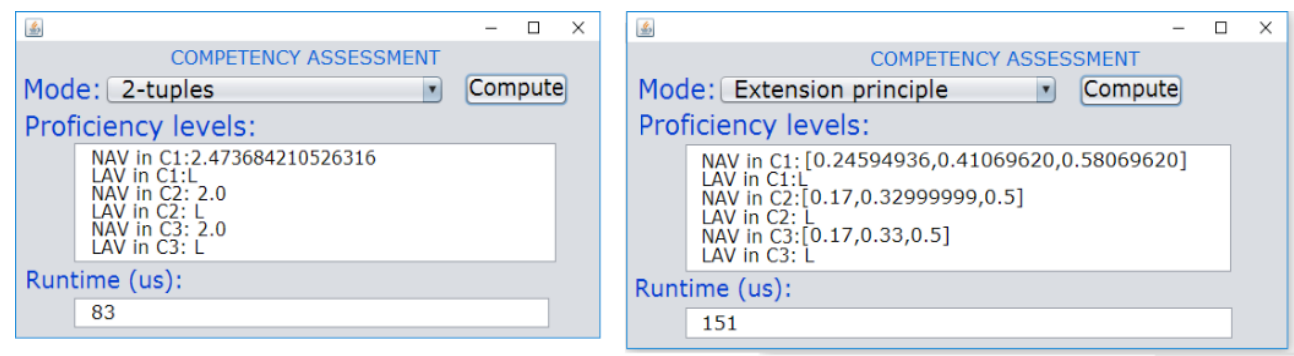

Figura 9 - Interfaces de la aplicación. A la izquierda se presenta la interfaz del modo 1 y a la derecha la del modo 2

Los experimentos y la colección de datos fueron realizados y obtenidos ejecutando las siguientes fases:

1. La estructura de los mapas fue adaptada al formato JSON y almacenada en la base de datos MongoDB. Cada estructura fue identificada por medio de un identificador único.

2. Los $\mathrm{NC}$ alcanzados en las $\mathrm{AE}$ fueron introducidos en la base de datos. Cada estudiante fue identificado con un identificador único.

3. Los NC para cada competencia (745 en total) de los mapas fue computada por la aplicación (usando el Modo 1 de la herramienta).

4. La fase 3 fue realizada utilizando el modelo basado en el principio de extensión (usando el Modo 2 de la herramienta).

5. Con el propósito de medir la efectividad de la propuesta, los NC dados por la aplicación en las fases 3 y 4 fueron comparados con los provistos por los docentes. Entonces, el porcentaje de aciertos fue medido mediante la expresión (6).

6. Con el propósito de conocer las ventajas de la presente propuesta en términos de tiempo de ejecución en comparación con el enfoque basado en el principio de extensión, todos los mapas fueron computados 100 veces para medir el tiempo de ejecución promedio de cada estructura y enfoque mediante la expresión (7). Cabe mencionar que los tiempos medidos fueron tomados para los métodos de la aplicación que están directamente relacionados a los procesos propios de cada enfoque (otros métodos como los relacionados con la gestión de bases de datos, interfaces, etc., no fueron considerados en las medidas). 
La Tabla 1 presenta los resultados alcanzados en los experimentos de la fase 5. Los datos están organizados de acuerdo con cada uno de los dos enfoques evaluados y para cada estructura de los mapas. Analizando los resultados, el porcentaje de aciertos es 98.39\% y 96.91\% respectivamente. Por consiguiente, la efectividad del modelo puede ser considerada como positiva. Para validar la significancia estadística de los resultados, se quiere comprobar si existe una diferencia significativa entre los resultados de los dos enfoques. Inicialmente se plantean las siguientes hipótesis:

\begin{tabular}{|c|c|c|c|c|c|c|}
\hline \multirow[b]{2}{*}{ Estructura } & \multirow[b]{2}{*}{$\begin{array}{l}\text { Competencias por } \\
\text { estructura }\end{array}$} & \multirow[b]{2}{*}{$\boldsymbol{T}$} & \multicolumn{2}{|c|}{ Propuesta } & \multicolumn{2}{|c|}{$\begin{array}{l}\text { Enfoque basado en el } \\
\text { principio de extensión }\end{array}$} \\
\hline & & & $\boldsymbol{N}_{\text {hits }}$ & $A_{1}(\%)$ & $N_{\text {hits }}$ & $A_{2}(\%)$ \\
\hline 1 & 8 & 40 & 39 & 97.5 & 36 & 90 \\
\hline 2 & 6 & 30 & 28 & 93.33 & 27 & 90 \\
\hline 3 & 15 & 75 & 72 & 96 & 71 & 94.66 \\
\hline 4 & 7 & 35 & 34 & 97.14 & 32 & 91.42 \\
\hline 5 & 11 & 55 & 54 & 98.18 & 53 & 96.36 \\
\hline 6 & 3 & 15 & 15 & 100 & 15 & 100 \\
\hline 7 & 11 & 55 & 55 & 100 & 55 & 100 \\
\hline 8 & 10 & 50 & 50 & 100 & 50 & 100 \\
\hline 9 & 24 & 120 & 119 & 99.16 & 119 & 99.16 \\
\hline 10 & 18 & 90 & 89 & 98.88 & 88 & $97 \cdot 77$ \\
\hline 11 & 3 & 15 & 15 & 100 & 15 & 100 \\
\hline 12 & 10 & 50 & 49 & 98 & 49 & 98 \\
\hline 13 & 3 & 15 & 15 & 100 & 15 & 100 \\
\hline 14 & 9 & 45 & 44 & 97.77 & 43 & 95.55 \\
\hline 15 & 11 & 55 & 55 & 100 & 54 & 98.18 \\
\hline Total & 149 & 745 & 733 & 98.39 & 722 & 96.91 \\
\hline
\end{tabular}

Tabla 1 - Resultado de aciertos

$H_{o}$ (Hipótesis nula): no hay diferencias significatias entre los dos enfoques; $H_{a}$ (Hipótesis alternativa): si existen diferencias significativas. Calculando una probabilidad de acierto promedio entre los enfoques se obtiene:

$$
p=\frac{A_{1}+A_{2}}{2}=\frac{0.9839+0.9691}{2}=0.976655
$$

Considerando un nivel de significancia $\alpha=0.05$, el valor estadístico crítico en la distribución normal es $Z_{\alpha=0.05}=1.96$. El error estándar es:

$$
e s=\sqrt{p(1-p)\left(1 / T^{+1} / T\right)}=0.007823
$$


Para concluir acerca de las hipótesis, se evalúa si $\left|A_{1}-A_{2}\right|=0.184$ es mayor o menor que $e^{*} Z_{\alpha=0.05}=0.0163$. Dado que $\left|A_{1}-A_{2}\right|$ es mayor que $e s^{*} Z_{\alpha=0.05}$, se concluye que la diferencia entre los aciertos del enfoque propuesto y los aciertos del basado en el principio de extensión es estadísticamente significativa. Por consiguiente, se rechaza $H_{o}$ y se acepta $H_{a}$ como probablemente cierta. Por otro lado, las medidas de tiempo de ejecución promedio para cada enfoque y estructura son presentadas en la Figura 10. Analizando los resultados, los tiempos de ejecución para el enfoque basado en el principio de extensión son mayores que los de la propuesta entre una y dos veces en el 40\% de los casos, y más de dos veces en el 60\% de los casos. Éstos resultados son acordes con la complejidad computacional inherente a cada enfoque. Mientras que en la propuesta el proceso computacional se realiza sobre un único valor $\beta$ que representa la cantidad de información de cada término lingüístico (gracias al uso de 2-tuplas), el enfoque basado en el principio de extensión obliga al uso constante de los 3 valores que constituyen la semántica de cada término. Esto presume un mayor consumo de memoria y procesador, lo cual explica las diferencias medidas en tiempos de ejecución entre cada enfoque.

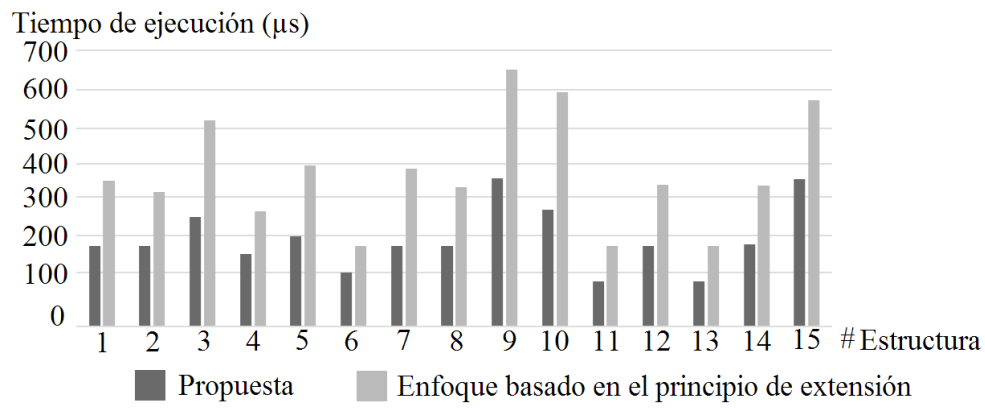

Figura 10 - Resultados de tiempos de ejecución

\section{Conclusiones y trabajos futuros}

La evolución de los sistemas computacionales implica cambios en el diseñoy evaluación de los procesos de enseñanza y aprendizaje. En este caso, se ha propuesto un enfoque difuso para evaluar competencias, basado en las características de los mapas de competencias. El enfoque computa los NC de todos los elementos del mapa usando los alcanzados en las $\mathrm{AE}$, permitiendo a estudiantes y docentes conocer las metas educativas alcanzadas en el currículo de un programa, área o curso. El enfoque ha sido implementado como una herramienta software Java y evaluado con datos reales de mapas provistos por docentes. Los resultados indican que es bastante efectivo, ofreciendo un porcentaje de aciertos cercano al 100\% y más alto que el del enfoque basado en el principio de extensión. Además, provee valores bajos de tiempo de ejecución, por lo cual, es viable para ser integrado a plataformas de e-learning o servicios de internet para apoyar procesos educativos reales.

En trabajos futuros será estudiada la forma para incorporar la presente propuesta a un sistema de recomendación de contenidos de VoD (Video bajo Demanda). Con el propósito de proveer perfiles de usuario, los NC lingüísticos pueden ser adaptados 
a una estructura de información (por ejemplo, matrices). Por lo tanto, el proceso de filtrado podría estar enfocado en las competencias no alcanzadas por los estudiantes. Adicionalmente, se buscará relacionar los contenidos con los mapas de competencias. En este sentido, el diseño de perfiles de aplicación de metadatos puede ser útil, adaptando diferentes esquemas como TV-Anytime, Learning Object Metadata (LOM), Resusable Competency Definition (RCD) y Simple Resusable Competency Map (SRCM).

\section{Agradecimientos}

Este trabajo es apoyado por el programa Doctorados Nacionales de Colciencias, convocatoria 617-2013.

\section{Referencias}

Fung, K. K. (2013). A Fuzzy Qualitative Evaluation System: A multi-granular aggregation approach using fuzzy compound linguistic variable. Journal of Intelligent \& Fuzzy Systems, 24, 61-78. doi:10.3233/IFS-2012-0531.

García, M., \& Gairín, J. (2011). Los Mapas de Competencias: una Herramienta para mejorar la Calidad de la Formación Universitaria. Revista Iberoamericana sobre Calidad, Eficacia y Cambio en Educación, 9(1), 84-102.

Herrera, E., López, A., Alonso, S., Moreno, J., Cabrerizo, F., \& Porcel, C. (2009). A computersupported learning system to help teachers to teach fuzzy information retrieval systems. Information Retrieval, 12, 179-200. doi:10.1007/s10791-008-9087-3.

Herrera, F., \& Martínez, L. (2000). A 2-Tuple Fuzzy Linguistic Representation Model for Computing with Words. IEEE Transactions on Fuzzy Systems, 8(6), 746-752. doi: 10.1109/91.890332.

Jia, Z., \& Zhao, L. (2010). Evaluation of Enterprise Core Competence Based on the Entropy and Triangular Fuzzy Number. In Proceeding of the International Conference on E-Business and E-Government (ICEE), 2045-2048. doi:10.1109/ ICEE.2010.517.

Likert, R. (1932). A technique for the measurement of attitudes. Archives of Psychology, 140, 1-55.

Massanet, S., Riera, J. V., Torrens, J., \& Herrera, E. (2014). A new linguistic computational model based on discrete fuzzy numbers for computing with words. Information Sciences, 248, 277-290. doi:10.1016/j.ins.2013.06.055.

Ministerio de Educación de Colombia. (2006). Estándares básicos de competencias. Retrieved from http://www.mineducacion.gov.co/1621/articles-340021_ recurso_1.pdf.

Montero, J., Alias, F., Badía, D., Fonseca, D., \& Vicent, L. (2014). A method for designing automatic assessment systems based on teachers reasoning for evaluating subjective engineering student's competences. In Proceedings of the 9th Iberian Information Systems and Technologies (CISTI). doi:10.1109/CISTI.2014.6877046. 
OCDE. (2006). El Programa PISA de la OCDE. Retrieved from http://www.oecd.org/ pisa/39730818.pdf.

Ostyn, C., (2006). Simple Reusable Competency Map. Retrieved from http://studylib. net/doc/18849165/simple-reusable-competency-map.

Pengshun, Z., \& Jianguo, H. (2012). Application of Fuzzy Hierarchy Evaluation in the Occupational Competency Assessment of the Vocational Colleges. In Proceedings of the 2012 International Symposium on Information Technology in Medicine and Education, 182-185. doi:10.1109/ITiME.2012.6291276.

Serrano, J., Romero, F., \& Olivas, J. (2013). Hiperion: A fuzzy approach for recommending educational activities based on the acquisition of competences. Information Sciences , 248, 114-129. doi:10.1016/j.ins.2013.06.009.

Zhang, H. (2012). The multiattribute group decision making method based on aggregation operators with interval-valued 2-tuple linguistic information. Mathematical and Computer Modelling, 56(1), 27-35. doi:10.1016/j.mcm.2012.01.001.

Zhaoa, X., \& Yueb, W. (2012). A multi-subsystem fuzzy DEA model with its application in mutual funds management companies' competence evaluation. Procedia Computer Science, 1, 2469-2478. doi:10.1016/j.procs.2010.04.279.

Zhaojun, Z., Xiao, Q., \& Xiaoqing, L. (2010). Competency Assessment Study Based on IPCA-Fuzzy Comprehensive Evaluation. In Proceedings of the International Conference on Management and Service Science (MASS). doi:10.1109/ ICMSS.2010.5578053. 